\begin{tabular}{c} 
International Journal of Engineering \& Technology, 7 (4) (2018) 2374-2378 \\
International Journal of Engineering \& Technology \\
SPC \\
Website: $\begin{array}{c}\text { ww. sciencepubco.com/index.php/IJET } \\
\text { doi: } 10.14419 / \text { ijet.v7it.14734 } \\
\text { Research paper }\end{array}$ \\
\hline
\end{tabular}

\title{
Enhanced approach for face detection and identifying human body proportionality using v-jones algorithm
}

\author{
Santosh Patil ${ }^{1}$ *, N. Ramakrishnaiah.2, S. Laxman Kumar ${ }^{3}$ \\ ${ }^{1}$ Assistant professor, Department of Computer Science and Engineering, \\ Institute of Aeronautical Engineering Hyderabad, Telangana 500043 \\ ${ }^{2}$ Professor, Department of Computer Science and Engineering University college of Engineering, \\ JNTUK Kakinada Andhaa Pradesh 533003 \\ *Corresponding author E-mail: santosh.p.shr@gmail.com
}

\begin{abstract}
Manual analysis of pedestrians and crowds is often impractical for massive datasets of surveillance videos. Automatic tracking of humans is one of the essential abilities for computerized analysis of such videos. In this proposed work we use Viola jones method for detecting moving human object, next using same method we identify the Human anatomy body proportion to detect the whole human body. The final function is the skin color threshold using the HIS and YCbCr. The proposed method yields high accuracy, we conducted experimental analysis on different videos, achieved high accuracy in detecting human object moment. Several future enhancements can be made to the system. The detection and tracking of multiple people can be extended to real-time live video. Apart from the detection and tracking, process of recognition can also be done.
\end{abstract}

Keywords: Tracking; Video Analysis; Threshold; HIS.

\section{Introduction}

Video is the most important element of the multimedia calculated and communiqué atmospheres. Many people used this technology by its easy thoughtful from inspiring by video advancement without time from capturing in the devices of digital media, collection, upload and downloading. Video analysis is the very important task in computer vision in human communication and it is a dangerous technology in the detection and tracking of human by measurement. The communications like artificial intelligence, computer visualization and graphics are related to humans in the area of video. In some cases like surveillance for confidence, biometric, animation, robotics for human interaction, vehicle detection are diligence for the reliable of detection and tracking of humans. The process such as human detection is the method for finding the presence of a person in image or video.

Human following is the procedure strategies for assessing video outline succession that speak to human development in the video and it needs a human discovery machine in each casing. The calculation put on essential indicator on information video success on taking into account viola jones course protest finder calculation, to identify human abdominal area and returns bouncing boxes that straightforwardly nourished into human life structures body extent to follow the head and face positions of people in individual video outlines.

The tracking and detection takes a human location with false positive, detection of the top of the body of human entity with detector. In particular, classify human or non-human environment and check the skin with color information and also detects the face from secondary skin by improving the pixel from the separation of skin and non-skin. Tracking is a process of complete repetition of detection method foe each dynamic changes in the video by progressive frame.

\subsection{Video body detection}

Human detection takes a amount of software application like façade tracing face identification and picture observation. Most detection algorithms Informed to be located at the detection of human face. Face detection is the necessary main step to direction of the many progressive computer visualization, biometric identification and software application. The characteristics of cascade object detector algorithm are:

- Robust

- Real time

- Face detection

The rectangular area is the quality required to the detection of background by including a number of image pixels. Here, Haar basis is a function, it has the similarity with the previously used detection with area based objects. The more general complex feature presented by "viola and jones" rely on with the more number of rectangular areas.

The drawback of this process is the motivation for discovering a front face with better light circumstances. Some other detectors has the proficient detection by easily detecting a top of the body as large objects. Viola-jones upper body model utilizes the features of haar function to translate in particular at the front part of the body in animal contains the face and brains and part of the body between the neck and upper arm area. This method is most successful and detects the human body by sitting, standing and walking in different illumination, position by front or the side view and also this method is steady by changing a position for example rotation or tilting of head. Every detection have their benefits and limitation. The main 
limitation of viola jones upper body detection is the high false positive rate.

Background subtraction, optical flow and spatiotemporal filtering approaches are the most predictable method for the detection of object. It provides good results by the multiple performance of apparent combination of the detections. The standard viola jones detector has the primary cascade object detector system, which uses the first step to detect the higher part in the body, which is the front part of the body in human that contain the face and the part of the body between the neck and the upper arm.

\subsection{Human anatomy body proportional}

A skeletal construction of human body is Anatomy It is a collection of muscles, skeleton, and proportionality. This is subtitles variance between individuals, human proportionality fit within a moderately standard scope, through creative people have historically tested to create idealized standard. Which have wide-ranging consider over different points and

areas. In from the tip top of the head to the momentum. This unit of mensuration is sensibly standard, and has long been used by creative people to launch the proportionality of the human.

\subsection{Skin color threshold}

Skin color is the separate characteristics of the human face. It has a standard parameter to the computer visualization to detect the human. The detection of skin is the calculation framework which is used to check and find the image with the region of face in the whole body of human. The feature of processing a color technique is used to check a partial number of pixel (This decrease the detection rate). The rod shaped photoreceptors located in the retina of human is not differentiate by color, but its brightness is most sensitive to the detection of human face.

\subsection{Review of the literature}

Viola jones method and skin detection combination of the using hybrid face detection system [1] proposed on the main impartial to rises the appearance of detection by organization in a face position with growing speed and less false rate of positive values in images with a background of complex nature based on the three hybrid detector algorithm. Viola jones use the primary detector upper body model for high possibility of find face in the region instead of searching the entire image. Secondary detector is used to find the inaccurate face in that region and increase accuracy and reduces false negatives by Viola jones face detector.

Viola jones and geometric use by the detect and analyse image parts information approache[2] intensive on improve fully computerized by the detection of human face to identify the organs of the face with complex background, and covers the advanced task localization and volume of the part with facial mental position by applying various methods in detection.

Kruppa $\mathrm{H}$ and Castrillon-santana M [3] describe a technique which is introduction for visual surveillance and presentation valuation of tracking and surveillance. The presentation of the detector of the accurateness and speed is estimated on data sets from pets database and compared to the thing centered method particular kindness os paid to the role of available image resolve.

Liu Zhu and Wang [4] propose on new approach for face detection and tracking in video are combined. The algorithm for face detection is fast pattern identical using the iterative dynamic programming.

Feris Rogerio Schmidt et. al [5] presents a tracking and detection of facemask with a structure in sequence of video at real time system. System used by graphical communication application like teleconferencing, practical reality, intelligent interface, human communication machine and surveillance. They use a model with statistical color of skin to fragment the region of face in a candidate at image. Based on the template of efficient matching scheme with each region in the absence of face which is confirmed by detector.
Fonseca Pedro Miguel et al[6] proposed on a object tracking algorithm with a compressed generic domain object with a combination of algorithm for the detection of face. The algorithm has a computational result with low cost and the problem is detected and tracked by a frame with a sequences of compressed video like "MPEG-1 or MPEG-2". Object like face can be tracking through video stream using motion info providing by current frontward and retrograde motion vector.

\section{Proposed method}

The system architecture diagram presents or describes the overview of entire proposed method. Describes the main components and the interfaces considered to develop the system. High level design uses no-technical to some technical terms that would be understood by the system. Figure 1, shows the planning system of the projected organization. The structure design diagram of the

proposed method mainly contains 3 modules. The three different modules are, video detection object, tracking selected portion of moving object and Human Anatomy Body Proportion final module is the Skin color Threshold. Input to the system is The read video or upload video. This video converted by the frame by frame to apply the algorithm viola jones upper detector by each frame to complete done by detector then is human anatomy body proportion this using calculate the height and width of the human body.

The next module or final module check the skin color for human only calculated face area in the represent the face using the color space in a accurate demonstration set of color video system CMYK used in color printing and true positive skin or false negative skin done by the skin color to track the human.

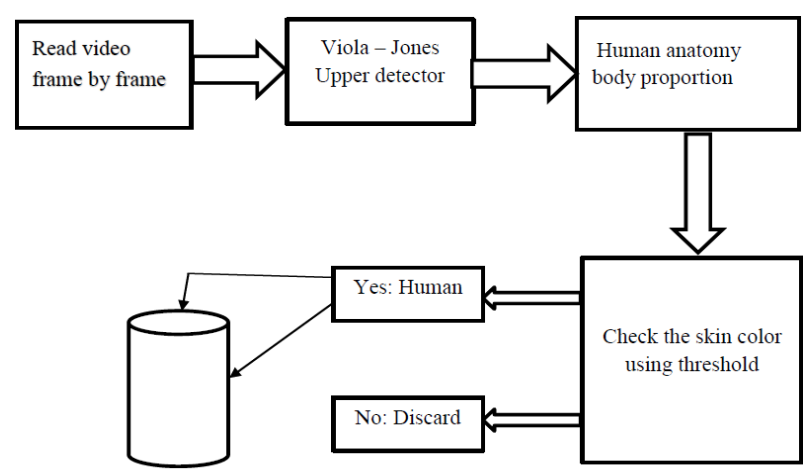

Fig. 1: System Architecture of Proposed Technique.

The analyser will input the video for processing. The function multiple people detection will detect the moving human objects in every frame by using Paul Viola Joes method. The next function Human anatomy body proportion using paul viola jones to detect the whole human body.The final function is the skin color threshold using the HIS and $\mathrm{YCbCr}$ means $\mathrm{Cb}$ is a chromo of the blue and $\mathrm{Cr}$ is a chromo is a red its every pixel from separation between skin pixel and non pixel and calculated the predictable façade area and translating RGB demonstration to chromo illustration. and defining the probability value founded on the calculation $140<\mathrm{Cr}<165 \& 140$ $<\mathrm{Cb}<195$ a section of the carroty to red to pink in red-difference and blue-difference channels $0.01<$ Hue $<0.1$ this means hue is basically reddish this define the skin pixel color.

\subsection{Video detection}

First module is the read by video. This video converted by the frame by frame and frame extracted this frame apply the cascade object detector algorithm uses the Haar feature to detect the face detection with bounding box and each every parts like eyes, mouth, nose with bounding box and then calculated the $\mathrm{x}, \mathrm{y}$ value of the face region and also detect the head region using this same algorithm.

If any case face positive or not detect properly go to discard the stop the detecting or restart the intial stage detect the face will start and then next method this is used by the calculated by the height and 
width of the human using the same algorithm and then next is skin color threshold this is check the color information enhanced separation between skin pixel non-skin pixel using the converting the RGB to HIS this function uses the finding the skin color threshold. If any case negative skin color go to the discard otherwise if positive check the skin color go to tracking the multiple people in movement with bounding box to whole body and next step is clustering. Clustering and grouping are the both important task in knowledge and accepting. Bundling is the organization of object into changed collections, or more exactly the dividing of a data set into cluster. This clustering using the $\mathrm{K}$ mean algorithm into several step include the $\mathrm{K}$ mean algorithm.

The result of the clustering algorithm apply the multiple people by mean value can be recognized. The best image for each people Tracking selected portion of moving object In video object detection first read the video frame by frame and finding the face in frame using haar cascade.to apply the algorithm viola jones object detector uses the Haar feature to encrypt the head and shoulder region.

Since it is using the further feature about the head, This model is the more strong the detect humanoid higher body walking, standup, and sitting for dissimilar lighting and dissimilar position front or side also the perfect is steady against posture changes, e.g. head turnings and were used in various significant applications such as visual surveillance, sports video analysis etc. Various numerical approaches have been suggested to model scene backgrounds. If found the face then go to next is find the eye, mouth and nose, using haar cascade.

The finally output is detecting the face in region with bounding box to apply the primary detector system making people differences among moving people in a video sequence.

\subsection{Human anatomy body proportion}

the human anatomy body proportion first step is the read video by frame by frame then find face using haar features cascade object detector and extract the face region to the people higher region .To detect the human upper body using the viola jones cascade object detector. If any case found the human upper body finally the to detect the human upper body with bounding box.

The face detection finally completed the next step is human anatomy body proportion using this same algorithm to calculated the human hight, width of the human to use of the best human position.

\subsection{Clustering}

Clustering plus grouping are the mutually important task in knowledge and accepting. Grouping are the organization of object into changed collections, or further exactly the dividing of a statistics set interested in cluster. This clustering using the $\mathrm{K}$ mean algorithm into several step include the $\mathrm{K}$ mean algorithm.

Clustering Old-style cluster methods are advanced to analyse whole documents collections. The clustering uses the k-means algorithm. Cluster is used to categorize or to collection $n$ items founded on qualities into $\mathrm{k}$ dividers the items founded on qualities/structures into a $\mathrm{K}$ amount of groups, where $\mathrm{K}$ is progressive number identified numeral.

The result of the clustering algorithm apply the multiple people by mean value can be recognized. The greatest image for everyone people is the calculated the width and height for highest for each cluster in the list which can be protected as greatest humanoid position.

\subsection{Pseudo code for viola jones method}

Paul Viola Jones method takes video frames as input and determines the non-stationary objects in every frame. The finally output is matrix has 4 item

// Input: image

//Output: Detected image

Function dctor $=$ bdctor $($ TFace, Tparts, Ssize $)$
IF $($ nar $<1)$

TFace 1

END

IF $($ nar $<2)$

Tparts 1

END

IF $($ nar $<3)$

Ssize 176

END

Name Dctor $\left\{\right.$ „Leye ${ }^{e e}$, ,Reye ${ }^{e e},$, ,mouth ${ }^{e e},$, ,nose $\left.{ }^{e e}\right\}$

Dctor. Ssize Ssize.

Dctor.Dctor cell;

FOR: for $\mathrm{k}=1$ to 4 .

Pseudo code for Detects face parts

The detects face parts using cascade object detector the proposed system to detect the face with bounding box and each every object detect

//Input: given input image from source

// Output: detect faces with parts

Function [Bbox,bbx,faces,bbfasec] DtctFaceparts(Dctor,x,thick)

IF $($ nar $<3)$

Set k 1

END

Detecting the Face

Call step function

Obtain size of boundary box.

Pad zeros to boundary box.

Detecting the parts

nd for $\left\{\right.$,Leye ${ }^{\text {ee }}$, ,Reye ${ }^{e e}$, ,mouth ${ }^{e e}$, ,nose $\left.{ }^{e e}\right\}$

Set mins.

Obtain Ssize as

Ssize dctor size

For K 1

Set region 1

ELSE if $\mathrm{k}=2$

Set region 2

ELSE if $\mathrm{K}=3$

Set region 3

ELSE if $K=4$

Compute rgn value by considering Ssize value

ELSE

Set rgn with Ssize value

END

Pad zeros to bsize

Compute the value of $x$

Relize the $\mathrm{x}$

IF ( size $b>0)$

Increment pnum.

Pnum=pnum +1

IF $\mathrm{k}=1$

Sort the rows and store it bsize

ELSE IF K = 2

Compute flipud for first Boundary points

ELSE IF k = 3

Compute flipud for second boundary point

ELSE IF k = 4

Compute flipud for third boundary point

ELSE IF k = 5

Compute the value 4 boundary points.

END

END

Compute the value of bbox.

Compute the sum of 4 boundary points

END

Draw the faces

Calculate value of box by taking box and part number as the parameter

Check whether part number is less than or equal to 2

IF $($ Tk $>=0)$

Compute Tx and Ty

Tx ceil (t) 
Ty flour ( $\mathrm{t}$ )

ELSE

Tx 0

Ty 0

END

Store the value of $\mathrm{X}$ in bx.

Faces

IF value of nar is greater than 2

Compute the value of faces and boxface

FOR I 1 to size

Compute the value of face and bbfaces

END

\section{Performance analysis}

Performance analysis for video 1

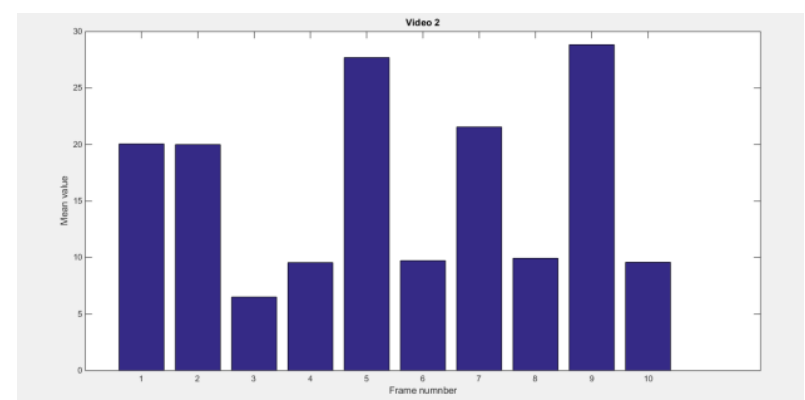

Fig. 2: Plot of Frame Number versus Mean Value for Video 1.

The above figure 2 show the plot of frame versus mean value ahd this mean value is multiple people tracking. The value $\mathrm{x}$ axis show the frame value respected videos and y axis show the mean value respected tracking value

Performance analysis for video2

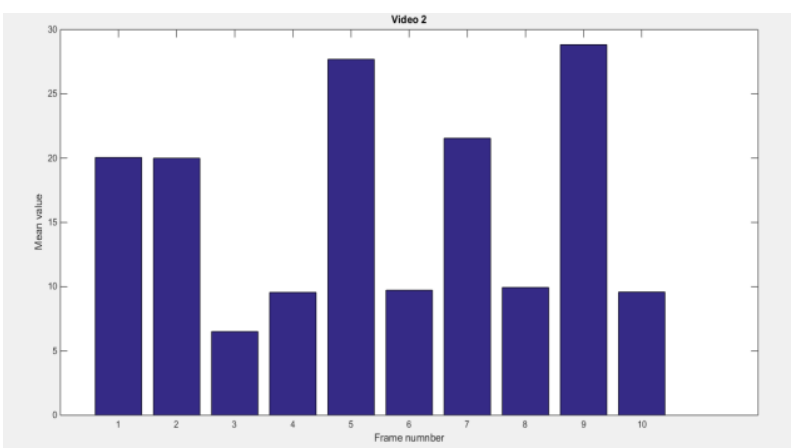

Fig. 3: Plot of Frame Number versus Mean Value for Video 2.

The figure 3 show the plot of frame versus mean value of the video 2 and this mean value is multiple people tracking. The value $\mathrm{x}$ axis show the frame value respected videos and $y$ axis show the mean value respected tracking value.

\section{Conclusions}

Video has turned into a critical component of sight and sound processing and correspondence situations. As the years pass by and individuals use innovation more it's anything but difficult to make great recordings in the blink of an eye because of the fast progressions in advanced gadgets gazing from catch, store, transfer and download. Human recognition and following are viewed as a basic innovation for machine/human communication and central undertakings of PC vision forms taking into account video examination. Wide video area identified with Human Computer Interaction (HCI, for example, PC design, manmade brainpower and $\mathrm{PC}$ vision. A few case of utilizations with solid human movement discovery and following are: observation for security, biometrics, creating characteristic activity, human collaboration for versatile apply autonomy, person on foot recognition on vehicles and programmed movement catch for video

The algorithm apply to the fast face detection and computing the human body proportionality from the upper body and calculating the face part area and check the color information enhanced separation between skin pixel and non skin pixel and movement multiple people tracking to calculated the height and width of the each human using clustering method this method using the $\mathrm{k}$ means algorithm. The final result is to calculated maximum height and width for human to decide best human position.

\section{References}

[1] A.Maghraby M.Abdalla O.Enany,Hybrid Face Detection System using Combination of Viola - Jones Method and Skin Detection, International Journal of Computer Applications , May 2013 ISBN : 973 93-80875-36-7.

[2] A.Maghraby M.Abdalla O.Enany, Detect and analyze face parts information using Viola-Jones and Geometric approaches, IJCA September 2014,ISBN : 973-93-8088364-3.

[3] Kruppa H., Castrillon-Santana M., and B. Schiele. "Fast and Robust Face Finding via Local Context". Proceedings of the Joint IEEE International Workshop on Visual Surveillance and Performance Evaluation of Tracking and Surveillance, 2003, pp. 157-164.

[4] Mahesh C. Pawaskar, N. S.Narkhede and Saurabh S. Athalye, "Detection of MovingObject Based On Background Subtraction"' in International Journal of Emerging Trends \& Technology in Computer Science, Vol. 3, Issue 3, pp. 215-218, May-June 2014.

[5] R. Manikandan, R. Ramakrishnan, "Human Object Detection and Tracking using Background Subtraction for Sports Applications", in International Journal of Advanced Research in Computer and Communication Engineering, Vol. 2, Issue 10, pp. 4077-4080, October 2013.

[6] Tang Sze Ling, Liang Kim Meng, Lim Mei Kuan, "Colour-based Object Tracking in Surveillance Application", in Proceedings of the International MultiConference of Engineers and Computer Scientists 2009 Vol I, March 2009, Hong Kong.

[7] C Raghavendra, 2A Kumaravel and P Anjaiah, " A New Hybrid Method For Image De-Noising In Light Of Wavelet Transform”, International Journal of Pure and Applied Mathematics, Vol. 116, No. 21, pp. 197-202, Oct. 2017

[8] Chatakunta Praveen Kumar, P Anyam Aditya Sharma, Y Harika Devi and K Radhika, "Implementation of Image Contrast Enhancement Using Automatic Threshold Selection and Histogram Equalization Techniques", Journal of Advanced Research in Dynamical and Control Systems, Vol. 9. Sp- 17, pp. 1159-1172, Dec.2017.

[9] Ch. Srikanth, MotheRakesh, and Y. SubbaRayudu "Enhanced Technique for Images to Control Visually Lossless JPEG2000", International Journal of Computer Sciences, Vol. 21, Feb. 2016.

[10] Valtteri Takala and Matti Pietikainen, "Multi-Object Tracking Using Color, Texture and Motion", in IEEE conference on Computer Vision and Pattern reorganization, pp. 1-7, Minneapoli 2007.

[11] Sunitha M.R, H.N Jayanna, Ramegowda, "Tracking Multiple Moving Object based on combined Color and Centroid feature in Video Sequence", in IEEE International Conference on Computational Intelligence and Computing Research, pp. 846-850, Dec 2014. https://doi.org/10.1109/ICCIC.2014.7238412.

[12] Priti P. Kuralkar , V.T.Gaikwad, "Human Object Tracking using Background Subtraction and Shadow Removal Techniques" in International Journal of Advanced Research in Computer Science and Software Engineering, Volume 2, Issue 3, March 2012.

[13] Sihwan Kim, Tadashi Shibata, "Feature-Based Object Tracking Using Spatial Matching of Differential Directional-Edge Images", in

[14] S.Bhuvaneswari, T.S.Subashini, "Tracking Manually Selected Object In Videos Using Color Histogram Matching", in Journal of Theoretical and Applied Information Technology, Vol. 67, Issues 3, pp. $562-568$, September 2014.

[15] M.Besita Augustin, Mrs. Sujitha Juliet, Mr. S. Palanikumar, "Motion and Feature Based Person Tracking In Surveillance Videos", in Proceedings Of ICETECT, Vol. 19, no. 7, pp. 780-785, Dec 2011.

[16] R. Venkatesh Babu, Anamitra Makur, "Kernel-Based Spatial-Color Modeling For Fast Moving Object Tracking" in IEEE International Conference on Acoustics, Speech, and Signal Processing, Vol 3, Issue 12, pp. 901-904, April 2007.

[17] Athilingam R, Mohamed Rasheed A, Senthil Kumar K, Kaviyarasu A, Thillainayagi, "Target Tracking with Background Modeled Mean 
Shift Technique for UAV Surveillance videos", in International Journal of Engineering and Technology, Vol 6,No 2 , pp. 805-814, AprMay 2014

[18] Kaihua Zhang, Lei Zhang, Ming-Hsuan Yang, "Real-Time Object Tracking via Online Discriminative Feature Selection" in IEEE Transactions on Image Processing, Vol. 22, No. 12, December 2013.

[19] N. Poornachandra Rao, R. Obulakonda Reddy and K. Tarakeswar, "Underwater Object Detection and Effective Classification Using Sparse Representation Vasershtein Metric", Jour of Adv Research in Dynamical \& Control Systems, Vol. 10, 01-Special Issue, 2018. 